\title{
2.5D Near-field Reproduction in Higher Order Ambisonics
}

\author{
Wen-Bei Wang \\ Speech and Audio Signal Processing Laboratory \\ School of Electronic Information and Control Engineering \\ Beijing University of Technology \\ Beijing 100124, China \\ wwb@emails.bjut.edu.cn \\ Mao-Shen Jia* \\ Speech and Audio Signal Processing Laboratory \\ School of Electronic Information and Control Engineering \\ Beijing University of Technology \\ Beijing 100124, China \\ jiamaoshen@bjut.edu.cn
}

\author{
Chang-Chun Bao \\ Speech and Audio Signal Processing Laboratory \\ School of Electronic Information and Control Engineering \\ Beijing University of Technology \\ Beijing 100124, China \\ baochch@bjut.edu.cn
}

\begin{abstract}
D) sound field reproduction is a hot topic in acoustic signal processing, which overcomes the dimensionality mismatch (DM) problem between 2dimensional (2D) sound field synthesis and practical sound sources. Based on the cylindrical/spherical harmonic expansion of Higher Order Ambisonics (HOA), a new method for 2.5D near-field reproduction is proposed in this paper. Firstly, a DM near-field corrected (DM-NFC) filter is proposed for restricting the $2.5 \mathrm{D}$ near-field distortion and achieving an efficient implementation. Secondly, we obtain the modified cylindrical harmonic expansion of the desired sound field based on cylindrical harmonic synthesis. Then, a general expression of the loudspeaker driving function is derived by using the concept of continuous loudspeaker array hypothesis. Finally, the proposed method is extended by using a circular array of 3-dimensional (3D) higher order (HO) loudspeakers, which provides a mode matching solution based on 3D wavefield translation. The test results indicate that the $2.5 \mathrm{D}$ near-field distortion is evidently eliminated by the proposed method. Furthermore, through using HO loudspeaker array, the proposed can significantly reduce the minimum number of loudspeakers for 2.5D near-field reproduction.
\end{abstract}

Keywords-Higher order Ambisonics, 2.5D near-field reproduction, DM-NFC filter, $3 D$ higher order loudspeaker.

\section{INTRODUCTION}

Sound field reproduction is a fundamental problem in acoustic signal processing. It aims to give one or more listeners the impression of being immersed in a realistic sound environment. Applications of this technique can be found in virtual auditory environments (VAEs) [1], such as home theater system, auditory display, and vehicle simulators. Sound field reproduction system pursues the physical synthesis of sound fields by driving a given ensemble of secondary (elementary) sound sources. The superposition of the sound fields radiated by the secondary sound sources produces a sound field over a certain region of space. At present, the practical choice of secondary sound sources follows the intrinsic dimensionality match principle. According to the psychoacoustic theory [2], the human ear is sensitive to the horizontal sound field at the same height. This establishes essentially the 2D reproduction problem, which is of interest in this paper. Based on the synthesis of the physical acoustics, the infinitely long line sound sources [3] should be replaced by $3 \mathrm{D}$ point sound sources which exhibit actual propagation of wave front in 2D sound field reproduction. Using 3D point sound sources (or loudspeakers) as secondary sound sources in 2D sound field reproduction, it is termed as the 2.5-dimensional (2.5D) sound field reproduction [4]. According to the position of virtual sound sources, a specific case of $2.5 \mathrm{D}$ sound field reproduction must be considered, which is that the virtual sound source is located within the loudspeaker array. This case is termed as $2.5 \mathrm{D}$ near-field reproduction.

The $2.5 \mathrm{D}$ near-field reproduction has many promising applications, such as the virtual classroom, vehicle simulators and multimedia conference call. However, the 2.5D near-field reproduction leads to the near-field distortion and the dimensionality mismatch problem. Hence, we propose a method that can handle this specific case in this paper, producing a virtual sound source located inside of an actual loudspeaker array.

So far, the implementation of 2.5D near-field reproduction mainly utilizes wave field synthesis (WFS) method [5-7] and higher order Ambisonics (HOA) approach [8-10]. The theoretical basis of WFS method is the Kirchhoff-Helmholtz integral equation (K-H integral). However, the aperture between loudspeakers is limited by the spatial sampling process. Meanwhile, the required number of loudspeakers is enormous over large areas. Recently, HOA has been related to the implementation of 2.5D sound field reproduction, where HOA is based on the cylindrical/spherical harmonic representations of sound fields. In these methods, 3D higher order loudspeakers [11] 
and $3 \mathrm{D}$ point sources are selected as the secondary sound sources. Nevertheless, most implementations of 2.5D HOA don't consider the near-field reproduction problem.

To address the reverberant issues, a new 2.5D near-field reproduction method for restricting the near-field distortion is proposed in this paper, by using a single circular 3D point sound source/3D higher order loudspeaker array. Firstly, based on cylindrical harmonic analysis of sound fields, the expansion representation of the 2D sound fields is obtained. Secondly, we propose a DM near-field corrected (DM-NFC) filter to restrict the near-field distortion. Further, the driving function of loudspeakers is designed by using the continuous loudspeaker concept. In addition, we also extend the $2.5 \mathrm{D}$ near-field reproduction to the simplification aspect of secondary sound sources by using 3D higher order loudspeaker array. Simulation results show that the proposed method can accurately synthesize the desired sound field and eliminate the near-field distortion evidently; meanwhile, the required number of loudspeakers is reduced by using $\mathrm{HO}$ loudspeakers.

The remainder of this paper is organized as follows. In Section 2, we present the proposed method of 2.5D nearfield reproduction. In Section 3, we extend the 2.5D nearfield reproduction to the simplification aspect of secondary sound sources. Finally, conclusions are drawn in Section 4.

\section{PROPOSED METHOD OF 2.5D NEAR-FIELD REPRODUCTION}

The HOA system employs cylindrical harmonic expansion [12] as a means to analyze 2D sound field. The theory of HOA method using cylindrical coordinates is based on the assumption that all sound sources lie beyond the loudspeaker array. However, when the sound sources are moved into the loudspeaker array, it can lead to near-field distortion which is caused by excessive energy components of cylindrical harmonics coefficients. In this section, we firstly get the cylindrical harmonic expansion of the 2D sound field. Secondly, a DM-NFC filter is proposed to eliminate excessive energy components. Finally, the loudspeaker driving function is designed.

\section{A. Cylindrical harmonic expansion of the desired sound field}

In cylindrical coordinates, the solution to the wave equation may be written in terms of cylindrical Bessel functions and cylindrical Hankel functions. The sound field generated by any number of virtual sound sources outside of the loudspeaker array, has the following representation:

$$
P_{d}(\boldsymbol{x}, k)=\sum_{m=-\infty}^{\infty} \underbrace{\frac{i}{4} H_{m}^{(2)}\left(k\left\|\boldsymbol{x}_{s}\right\|\right) e^{-i m \phi_{s}}}_{\alpha_{m}(k)} J_{m}(k r) e^{i m \phi}
$$

where $\operatorname{Pd}(\mathrm{x}, \mathrm{k})$ denotes the acoustic pressure at an observation point $\mathrm{x}=(\mathrm{r}, \phi)$. $\mathrm{xs}=(\mathrm{rs}, \phi \mathrm{s})$ is the position of virtual sound source and $\mathrm{k}$ is the wavenumber satisfied $\mathrm{k}=2 \pi \mathrm{f} / \mathrm{c}$, where $\mathrm{f}$ is the frequency and $\mathrm{c}$ is the speed of sound. $\alpha_{m}(k)$ are the interior harmonic expansion coefficients, and $\operatorname{Jm}(\bullet)$ is the mth order Bessel function of the first kind. The corresponding expansion for exterior sound field to any virtual sound sources is:

$$
P_{d}(\boldsymbol{x}, k)=\sum_{m=-\infty}^{\infty} \underbrace{\frac{i}{4} J_{m}\left(k\left\|\boldsymbol{x}_{s}\right\|\right) e^{-i m \phi_{s}}}_{\beta_{m}(k)} H_{m}^{(2)}(k r) e^{i m \phi}
$$

where $H_{m}^{(2)}(k r)$ is the cylindrical Hankel function of the second kind and $\beta_{m}(k)$ are the exterior expansion coefficients.

Based on the properties of the Bessel function within the region of interest which is a circular region of radius $\mathrm{r} 0$, (1) (2) can be truncated to $M$ terms as:

$$
P_{d}(\boldsymbol{x}, k)= \begin{cases}\sum_{m=-M}^{M} \frac{i}{4} H_{m}^{(2)}\left(k\left\|\boldsymbol{x}_{s}\right\|\right) e^{-i m \phi_{s}} J_{m}(k r) e^{i m \phi} \quad \text { interior sound field, } \quad\left(r \leq r_{0}\right) \\ \sum_{m=-M}^{M} \frac{i}{4} J_{m}\left(k\left\|\boldsymbol{x}_{s}\right\|\right) e^{-i m \phi_{s}} H_{m}^{(2)}(k r) e^{i m \phi} \quad \text { exterior sound field, }\left(r>r_{0}\right)\end{cases}
$$
2.718 .

where the truncation order $M=\left\lceil e r_{0} k / 2\right\rceil, \mathrm{e}=\exp (1)=$

\section{B. The dM-NFC filter}

According to the near-field synthesis [13], the interior expansion is invalid for the region $r>r 0$. Hence, when the interior expansion coefficients $\alpha_{m}(k)$ are used to synthesize the sound source within the loudspeaker array of radius $\mathrm{rl}$, the reproduced sound field diverges into the region $r>r 0$. This phenomenon is called as near-field distortion. A closer look at the properties of (3) for the region $r>r 0$ shows that it is actually the higher order components which introduce a high amount of energy at low frequencies into cylindrical harmonics coefficients [14]. To restrict the near-field distortion, the higher order harmonics need to be smoothly faded out. Hence, in order to achieve such an efficient implementation, a DM-NFC filter is proposed as follows:

$$
w_{m}^{D M-N F C}(k)=\frac{\mu_{2}}{\left\|H_{m}^{D M-N F C}\left(k, r_{s}, r_{l}\right)\right\|+\mu_{1}}
$$

where ${ }^{\mu_{1}}$ and ${ }^{\mu_{2}}$ are correction coefficients that need to be satisfied $\mu_{2}=1+\mu_{1} . H_{m}{ }^{D M-N F C}\left(k, r_{s}, r_{l}\right)$ represents the transfer function between 2D sound field and the secondary sound sources, which can be is expressed by:

$$
H_{m}^{D M-N F C}\left(k, r_{s}, r_{l}\right)=\frac{H_{m}^{(2)}\left(k r_{s}\right) h_{0}^{(2)}\left(k r_{l}\right)}{H_{0}^{(2)}\left(k r_{s}\right) h_{m}^{(2)}\left(k r_{l}\right)}
$$

By applying the proposed DM-NFC filter (4) to the cylindrical harmonic expansion of the interior sound field 
(3), we can get the modified expression of the $2 \mathrm{D}$ sound field as:

$$
P_{d}(\boldsymbol{x}, k)=\sum_{m=-M}^{M} w_{m}^{D M-N F C}(k) \frac{i}{4} H_{m}^{(2)}\left(k r_{s}\right) e^{-i m \phi_{s}} J_{m}(k r) e^{i m \phi}
$$

\section{Derivation of the secondary source driving function}

The key issue of 2.5D near-field reproduction is to design the loudspeaker driving function. Hence, this section will show the derivation of analytical expressions for the loudspeaker driving function using spherical harmonic analysis.

The reproduced sound field for a continuous circular distribution of secondary $3 \mathrm{D}$ sound sources centered on the two-dimensional Cartesian coordinates is given by:

$$
P_{r}(\boldsymbol{x}, k)=\int_{0}^{2 \pi} G\left(\boldsymbol{x}^{\prime}, k\right) T_{3 D}\left(\boldsymbol{x}-\boldsymbol{x}^{\prime}, k\right) r^{\prime} d \phi^{\prime}
$$

where $\boldsymbol{x}^{\prime}=\left(r^{\prime}, \phi^{\prime}\right)$ represents the secondary sound source (loudspeaker) position. $G\left(\boldsymbol{x}^{\prime}, k\right)$ denotes the driving function as:

$$
\begin{aligned}
& G\left(\boldsymbol{x}^{\prime}, k\right)= \sum_{m=-\infty}^{\infty} \xi_{m}(k) e^{i m \phi^{\prime}} \\
& \text { (8) } \begin{array}{l}
\text { equate the reproduced sound } \\
\text { desired sound field (6), i.e. , }
\end{array} \\
& w_{m}^{D M-N F C}(k) \frac{i}{4} H_{m}^{(2)}\left(k r_{s}\right) e^{-i m \phi_{s}} J_{m}(k r)=\sum_{n=-M}^{M}-2 \pi i k \xi_{m}(k) e^{-i m \phi} A_{n m}^{2} P_{n m}^{2}(\pi / 2) h_{n}^{(2)}\left(k\left\|\boldsymbol{x}_{l}\right\|\right) j_{n}(k r)
\end{aligned}
$$

where $\xi_{m}(k)$ is the mth Fourier expansion coefficient. Due to the fact that $x^{\prime}$ and $\mathrm{x}$ are in the same horizontal plane, the elevation of $x$ equals to that of $\boldsymbol{x}^{\prime}$ (i.e., $\pi / 2$ ). Based on the addition property of the spherical Hankel function, the loudspeaker $T_{3 D}\left(\boldsymbol{x}-\boldsymbol{x}^{\prime}, k\right)$ can be further represented by:

$$
T_{3 D}\left(\boldsymbol{x}^{\prime}-\boldsymbol{x}, k\right)=\sum_{n=0}^{\infty} \sum_{m=-n}^{n}-i k j_{n}(k r) h_{n}^{(2)}\left(k\left\|\boldsymbol{x}^{\prime}\right\|\right) A_{n m}^{2} P_{n m}^{2}(\pi / 2) e^{-i m \phi^{\prime}} e^{i m \phi}
$$

By substituting (8) and (9) into (7), we get the expression of the reproduced sound field as:

$$
P_{r}(\boldsymbol{x}, k)=\sum_{n=0}^{\infty} \sum_{m=-n}^{n}-2 \pi i k \xi_{m}(k) A_{n m}^{2} P_{n m}^{2}(\pi / 2) j_{n}(k r) h_{n}^{(2)}\left(k\left\|\boldsymbol{x}^{\prime}\right\|\right) e^{i m \phi}
$$

Based on Shannon sampling theorem, we can exactly reproduce $G\left(\boldsymbol{x}^{\prime}, k\right)$ by its samples. Since the mode of the desired sound field is limited to $M$, the (8) is truncated to $\mathrm{M}$ and the required number (noted $\mathrm{L}$ ) of loudspeakers is $2 \mathrm{M}+1$. To obtain the loudspeaker driving function, we equate the reproduced sound field (10) to the modified
In (11), given a certain $r$, a fixed set of Fourier expansion coefficient $\xi_{m}(k)$ can be calculated. To obtain the optimal loudspeaker driving function, the operation that sets $\mathrm{r}$ tend to zero has been adopted in the $2.5 \mathrm{D}$ far-away field analysis [4]. Note that the summation over $\mathrm{n}$ (11) reduces to a single term with $\mathrm{n}=\|\mathrm{m}\|$.

Then we get the Fourier series expansion coefficients of the driving function as:

$$
\xi_{m}(k)=\frac{w_{m}^{D M-N F C}(k) \frac{i}{4} H_{m}^{(2)}\left(k r_{s}\right) e^{-i m \phi_{s}} E_{m}}{-2 \pi i k A_{\|m\| m}^{2} P_{\|m\| m}^{2}(\pi / 2) h_{\|m\|}^{(2)}\left(k\left\|\boldsymbol{x}_{l}\right\|\right)}
$$

where

$$
E_{m}=\lim _{r \rightarrow 0} \frac{J_{\|m\| n}(k r)}{j_{m}(k r)}=\frac{\left(\operatorname{sgn}(m)^{\|m\|}\right)(2\|m\|+1) ! !}{2^{\|m\|}|| m \| !}
$$

By substituting (12) into (8), the loudspeaker driving function can be represented as:

$$
G\left(\boldsymbol{x}_{l}, k\right)=\sum_{m=-M}^{M} \frac{w_{m}^{D M-N F C}(k) \frac{i}{4} H_{m}^{(2)}\left(k r_{s}\right) e^{-i m \phi_{s}} E_{m} e^{i m \phi_{l}}}{-2 \pi i k A_{\|m\| m}^{2} P_{\|m\| m}^{2}(\pi / 2) h_{m}^{(2)}\left(k\left\|\boldsymbol{x}_{l}\right\|\right)}
$$

\section{Simulation results}

In order to evaluate the performance of the proposed method, two simulation experiments are considered in this subsection. Firstly, we investigate the performance of the proposed method for reproducing sound field. Secondly, we evaluate the reproduction errors of sound field for various frequencies. In these experiments, L loudspeakers are used, which are equally distributed in a circular array of radius $\mathrm{rl}$ $=1.5 \mathrm{~m}$. The HOA method without using the DM-NFC filter for 2.5D near-field reproduction is employed as the referenced approach.

The first experiment: The simulation is used to demonstrate the effectiveness of the proposed method. For convenience of the illustration purposes, the virtual sound source located at $(1, \pi / 4)$ is used, but the proposed method is not limited to such a position. The frequency of the selected sound source is $800 \mathrm{~Hz}$. The rule of thumb suggests that using the truncation order of sound field is $\mathrm{M}=21$; thus to avoid spatial aliasing, the minimum requirement number of 
loudspeakers is $\mathrm{L}=2 \mathrm{M}+1=43$. The reproduced sound fields by the referenced method and the proposed method are shown in Fig. 1. Listening area is enclosed by a black circle in each figure. The loudspeakers are shown as " $\mathrm{x}$ " marks. By comparing Fig. 1 (a) with Fig. 1 (b), it can be observed that the reproduced sound field within the interest region (the black circle) is almost identical with the referenced method. Nevertheless, the reproduced quality of sound field between the interest region and the loudspeaker array (the red circle) is significantly better than the referenced method. In addition, it can be observed that the quality of the reproduced sound field is also obviously better than the referenced method outside loudspeaker array. These simulation results validate that the proposed method can effectively eliminate the near-field distortion.

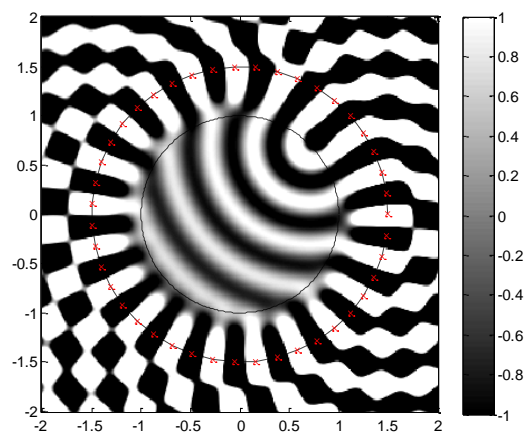

(a) without DM-NFC filter

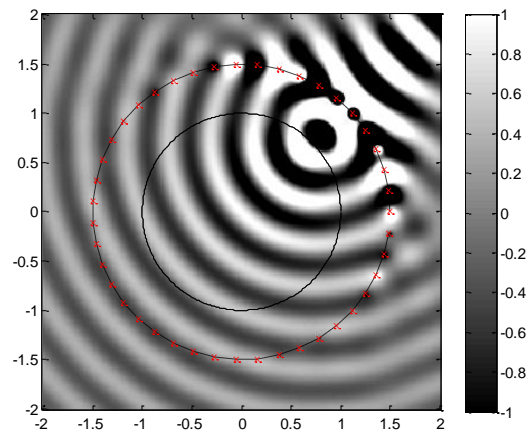

(b) with DM-NFC filter

Figure 1. Reproduction of a 2D cylindrical wave from the sound source located at $(1, \pi / 4)$, frequency $\mathrm{f}=800 \mathrm{~Hz}$.

The second experiment: Additionally, in order to evaluate the reproduction performance of the proposed method for different frequencies, the normalized mean square error (NMSE) is also computed. The NMSE (dB) is defined as follows:

$$
\varepsilon_{M(\mathrm{~dB})}(\boldsymbol{x}, k)=10 \log _{10} \frac{\int_{r_{1}}^{r_{2}} \int_{0}^{2 \pi}\left\|P_{d}(\boldsymbol{x}, k)-P_{r}(\boldsymbol{x}, k)\right\|^{2} r d \phi d r}{\int_{r_{1}}^{r_{2}} \int_{0}^{2 \pi}\left\|P_{d}(\boldsymbol{x}, k)\right\|^{2} r d \phi d r}
$$

According to (15), two more general representations of the reproduction performance are given in Fig. 2.

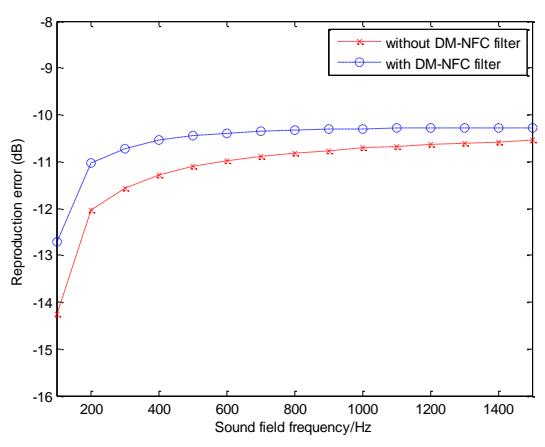

(a) interior field

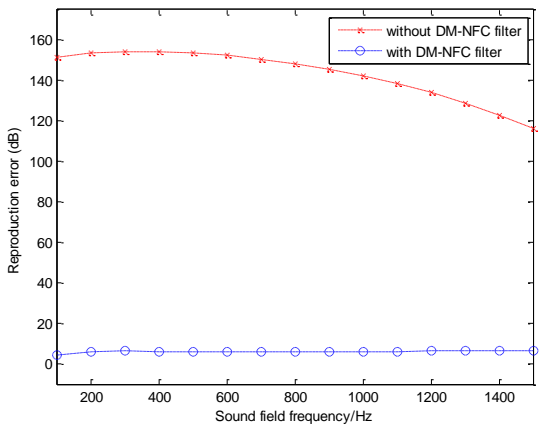

(b) exterior field

Figure 2. Interior field/exterior field reproduction errors as function of frequency $\mathrm{f}$ for two methods.

The Fig. 2 (a) compares the reproduction errors of the proposed method and the referenced method in the interior field (i.e., the NMSE $(\mathrm{dB})$ of the two methods in the area Dinterior $=\{\mathrm{x}(\mathrm{r}, \phi) \mid \mathrm{r}<\mathrm{r} 0\})$. Furthermore, the Fig. 2 (b) describes the reproduction errors of the two methods in exterior sound field (i.e., the NMSE (dB) of two methods in the area Dexterior $=\{\mathrm{x}(\mathrm{r}, \phi) \mid \mathrm{r} 0<\mathrm{r}<\mathrm{rl}\})$.

In Fig. 2 (a), it can be seen that the proposed method can provide accurate reproduction result in full frequency band. Meanwhile, the reproduction errors of the proposed method are almost close to the referenced method. Therefore, within the interior field, the desired sound field is accurately reproduced by the proposed method. From Fig. 2 (b), it can be observed that the reproduction errors of the proposed method are significantly lower than the referenced approach in exterior sound field. From these simulations, we can find that the proposed method eliminates near-field distortion and reproduces exterior sound field. In conclusion, it is worth recalling that the proposed method is more appropriate for $2.5 \mathrm{D}$ near-field reproduction.

\section{EXTENSION TO REPRODUCTION USING HIGHER ORDER LOUDSPEAKER}

In previous section, we have assumed that the $2.5 \mathrm{D}$ nearfield reproduction can be performed by the $3 \mathrm{D}$ point sound source array. However, this is not feasible in practice; meanwhile, for high frequencies over large areas in 2.5D HOA, the minimum numbers of loudspeakers to accurately 
reproduce the 2D sound field are enormous. Hence, in order to reduce the number of loudspeakers, we adopt the higher order loudspeaker array to reproduce 2D sound field in this section.

\section{A. 2.5D Near-field reproduction by higher order Loudspeaker Array}

In the spherical coordinates, given an observation point $\boldsymbol{x}=(r, \pi / 2, \phi)$, a 3D higher order (HO) loudspeaker positioned at $\boldsymbol{x}_{l}=\left(r_{l}, \pi / 2, \phi_{l}\right)$ of a circular region in the horizontal plane will radiate a sound field as:

$$
P_{l}(\boldsymbol{x}, k)=\sum_{\hat{m}=-\hat{N}}^{\hat{N}} \sum_{n=\|\hat{n}\|} \frac{w_{\hat{n}, \hat{m}}^{(l)}(k)}{h_{\hat{n}}^{(2)}\left(k r^{*}\right)} h_{\hat{n}}^{(2)}\left(k\left\|\boldsymbol{x}-\boldsymbol{x}_{l}\right\|\right) Y_{\hat{n}, \hat{m}}\left(\hat{\boldsymbol{y}}_{l}\right)
$$

where $\hat{N}$ is the order of the HO loudspeaker and $w_{\hat{n}, \hat{m}}(k)$ are complex amplitudes which represent the modal weights. $h_{\hat{n}}^{(2)^{\prime}}\left(k r^{*}\right)$ denotes first-order derivative of spherical Hankel function of the second kind, and $\mathrm{r}^{*}$ is the radius of the HO loudspeaker. $\hat{\boldsymbol{y}}_{l}=\left(\pi / 2, \bar{\phi}_{l}\right)$ is the direction vector of $\mathrm{yl}$, and $\mathrm{yl}=\mathrm{x}-\mathrm{xl}$.

$$
Y_{\hat{n}, \hat{m}}\left(\hat{\boldsymbol{y}}_{l}\right)=(-1)^{\hat{m}} A_{\hat{n}, \hat{m}} P_{\hat{n},\|\hat{m}\|}(\cos (\pi / 2)) e^{i \hat{m} \bar{\phi}_{l}}
$$

We employ $\mathrm{L}$ number of such $\mathrm{HO}$ loudspeakers in a circular discrete array, where the lth $(1=1,2, \ldots, \mathrm{L})$ loudspeaker is located at $\boldsymbol{x}_{l}=\left(r_{l}, \pi / 2, \phi_{l}\right)$. Hence, the reproduced sound field can be represented as:

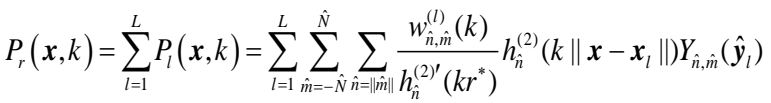

According to the spherical addition theorem for spherical Hankel function, we can get the reproduced sound field as:

$$
P_{r}(\boldsymbol{x}, k)=\sum_{m=-M}^{M} \sum_{n=\|m\|}^{M} \sum_{l=1}^{L} \sum_{\hat{m}=-\hat{N}}^{\hat{N}} \sum_{\hat{n}=\|\hat{m}\|}(-1)^{m} \frac{w_{\hat{n}, \hat{m}}^{(l)}(k)}{h_{\hat{n}}^{\prime}\left(k r^{*}\right)} S_{\hat{n}, n}^{n, m}\left(\boldsymbol{x}_{l}\right) j_{n}(k r) A_{n, m} P_{n,\|m\|}(\cos (\pi / 2)) e^{i m \phi}
$$

If $w_{\hat{n}, \hat{m}}^{(l)}(k)$ satisfy the condition that (19) equals to (6), the $\mathrm{HO}$ loudspeaker array can exactly reproduce desired sound field at all observation points $\mathrm{r}<\mathrm{rl}$ :

The reproduced sound field by referenced method and proposed method are shown in Fig. 3. By comparing Fig. 3 (a) with Fig. 3 (b), it can be observed that an apparent performance improvement by the proposed method. Within the interest region, the reproduced sound field corresponds well to the desired sound field. Meanwhile, outside the interest region, the proposed method also produces the best

$$
P_{r}(\boldsymbol{x}, k)=P_{d}(\boldsymbol{x}, k)
$$

\section{B. Simulation results}

In order to evaluate the performance of the proposed scheme, the results of the reproduced sound field are exhibited in this subsection. In these experiments, L HO loudspeakers are used, which are equally distributed on a circular array of radius $\mathrm{rl}=1.5 \mathrm{~m}$. In this experiment, for convenience of the illustration purposes, the setting conditions of simulation process are same to the above section. The orders of HO loudspeakers are set at $\hat{N}=1$. The minimum requirement number of $\mathrm{HO}$ loudspeakers is $\mathrm{L}$

$$
(2 M+1)
$$

$=\overline{(2 \hat{N}+1)}+\xi$. To ensure the best reproduction performance, $\xi$ is set at 3 in this paper.

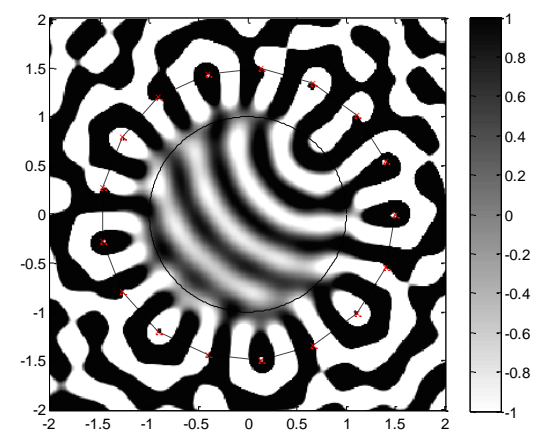

(a) without DM-NFC filter

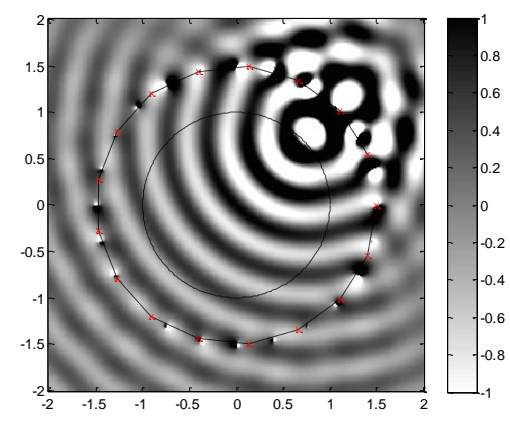

(b) with DM-NFC filter

Figure 3. Reproduction of a 2D cylindrical wave from the sound source located at $(1, \pi / 4)$, frequency $f=800 \mathrm{~Hz}$.

reproduction performance. The simulation again validates that the proposed method can evidently eliminate near-field distortion; meanwhile, compared to the point sound source array, the secondary HO loudspeaker distribution can significantly reduce the minimum number of loudspeakers. 


\section{CONCLUSION}

This paper proposes a new method for 2.5D near-field reproduction. Firstly, the modified expansion representation of sound field is obtained by the proposed DM-NFC filter. Then, a general expression of the loudspeaker driving signal is derived by using the concept of continuous loudspeaker array hypothesis. Finally, the proposed method is extended by using a circular HO loudspeaker array, which provides a mode matching solution based on $3 \mathrm{D}$ wavefield translation.

\section{ACKNOWLEDGMENTS}

This work has been supported by the Project supported by Beijing Postdoctoral Research Foundation, the National Natural Science Foundation of China (No. 61231015, 61201197).

\section{REFERENCES}

[1] Jia, M.S., Yang, Z.Y., Bao, C.C., Zheng, X.G., Ritz C.: Encoding multiple audio objects using intra-object sparsity. IEEE/ACM Transactions on Audio Speech and Language Processing, 23, 1082-1095 (2015).

[2] Brungart, D., Rabinowitz, W.: Auditory localization of nearby sources: Head-related transfer functions. The Journal of the Acoustical Society of America, 106, $1465--1479$ (1999).

[3] Bu, B., Abhayapala, T. D., Bao, C.C., Zhang, W.: Para-meterization of the three-dimensional room transfer function in horizontal plane. The Journal of the Acoustical Society of America, 138, 280--286 (2015).

[4] Zhang, W., Abhayapala, T. D.: 2.5D Sound Field Reproduction in Higher Order Ambisonics. In: 14th International Workshop on Acoustic Signal Enhancement (IWAENC), pp. 342-346. IEEE Press, Juan-les-Pins (2014).
[5] Wierstorf, H., Geier, M., Raake, A., Spors, S.: Perception of focused sources in wave field synthesis. Journal of the Audio Engineering Society, 61, 5--16 (2013).

[6] Spors, S.: Extension of an analytic secondary source selection criterion for wave field synthesis. International Journal of Tuberculosis \& Lung Disease, 13, 820--828 (2007).

[7] Kawano, T., Goto, K., Ishihara, T.: Analysis of scattered fields by an impedance discontinuity of a planar surface by using helmholtzkirchhoff integral theorem. Antennas and Propagation Society International Symposium (APSURSI), 9, 1--2 (2012).

[8] Choi, J.W., Kim, Y.H.: Sound field reproduction of a virtual source inside a loudspeaker array with minimal external radiation. IEEE Transactions on Audio Speech \& Language Processing, 21, 247--259 (2013).

[9] Choi, J.W., Kim, Y.H.: Integral approach for reproduction of virtual sound source surrounded by loudspeaker array. IEEE Transactions on Audio Speech \& Language Processing, 20, 1976--1989 (2012).

[10] Favrot, S., Buchholz, J.M.: Reproduction of nearby sound sources using higher-order ambisonics with practical loudspeaker arrays. Acta Acustica United with Acustica, 98, 48--60 (2012).

[11] Jia, M.S., Wang, W.B.: 2.5D Sound Field Reproduction Using Higher Order Loudspeakers. Cybernetics and information technologies, 15, 5--15 (2015).

[12] Wu, Y.J., Abhayapala, T.D.: Theory and design of soundfield reproduction using continuous loudspeaker concept, IEEE Transactions on Audio Speech \& Language Processing, 17, 107-116 (2009).

[13] Ahrens, J., Spors, S.: Focusing of virtual sound sources in higher order ambisonics. In: 124th Audio Engineering Society Convention, pp. 342-346. AES Press, Amsterdam, the Netherlands (2008).

[14] Daniel, J., Nicol, R., Moreau, S.: Further investigations of high order ambisonics and wavefield synthesis for holophonic sound imaging. In: 114th Audio Engineering Society Convention, pp. 1--18. AES Press, Amsterdam, the Netherlands (2003). 